\title{
Left Multiplicative Generalized Jordan Derivations of Semiprime Rings
}

\section{Reddy CJS ${ }^{1 *}$, Nagesh $\mathrm{K}^{2}$ and Kumar $\mathrm{AS}^{2}$}

${ }^{1}$ Department of Mathematics, Sri Venkateshwara University, Tirupati-517502, Andhra Pradesh, India

${ }^{2}$ Research Scholar, Rayalaseema University, Kurnool, Andhra Pradesh, India

\begin{abstract}
In this paper we prove that left multiplicative generalized Jordan derivation and left multiplicative generalized Jordan triple derivation of 2-torsion free semiprime rings are left multiplicative generalized derivation.
\end{abstract}

\section{Preliminaries}

Throughout this paper $R$ will be denote an associative ring with the center $Z(R)$. If $n>1$, a ring $R$ is said to be $n$-torsion free, if for $x \in R$, $n x=0$ implies $x=0$. Recall that a ring $R$ is called prime if for any $x, y \in R$, $x R y=\{0\}$ implies that either $x=0$ or $y=0$. And $R$ is a semiprime if $x R x=\{0\}$ implies $x=0$. An additive mapping $T: R \rightarrow R$ is said to be a left centralizer if $T(x y)=T(x) y\left(\operatorname{resp} . T\left(x^{2}\right)=T(x) x\right)$, for all $x, y \in R$. An additive mapping $T: R \rightarrow R$ is said to be a right centralizer if $T(x y)=y T(x)\left(\operatorname{resp} . T\left(x^{2}\right)=x T(x)\right)$, for all $x, y \in R$. An additive mapping $D: R \rightarrow R$ is called a derivation (resp. Jordan derivation) if $D(x y)=D(x) y+x D(y)$ (resp. $D\left(x^{2}\right)=D(x) x+x D(x)$ ), for all $x, y \in R$. An additive mapping $D: R \rightarrow R$ is called a left derivation (resp. Jordan left derivation) if $D(x y)=x D(y)+y D(x)$ (resp. $D\left(x^{2}\right)=x D(x)$ $+x D(x)$ ), for all $x, y \in R$. A mapping $F: R \rightarrow R$ is called centralizing on $S$ if $[f(x), x] \in Z$ for all $x \in S$ and is called commuting on $S$ if $[F(x), x]=0$ for all $x \in S$. An additive mapping $F: R \rightarrow R$ is called a generalized derivation if there exists a derivation $D: R \rightarrow R$ such that (resp. generalized Jordan derivation) $F(x y)=F(x) y+x D(y)\left(\right.$ resp. $\left.F\left(x^{2}\right)=F(x) x+x D(x)\right)$, for all $x, y \in R$. An additive mapping $F: R \rightarrow R$ is called a left generalized derivation if there exists a derivation $D: R \rightarrow R$ such that (resp. left generalized Jordan derivation) $F(x y)=x F(y)+D(x) y$ (resp. $\left.F\left(x^{2}\right)=x F(x)+D(x) x\right)$, for all $x, y \in R$. An additive mapping $D: R \rightarrow R$ is called Jordan triple derivation if $D(x y x)=D(x) y x+x D(y) x+x y D(x)$,for all $x, y \in R$. An additive mapping $F: R \rightarrow R$ generalized Jordan triple derivation if $F(x y x)=F(x) y x+x D(y)$ $x+x y D(x)$, for all $x, y \in R$ where $D$ is a Jordan triple derivation. An additive mapping $F: R \rightarrow R$ left multiplicative generalized Jordan triple derivation if $F(x y x)=x y F(x)+D(x) y x+x D(y) x$, for all $x, y \in R$ where $D$ is a Jordan triple derivation.

\section{Introduction}

Bresar [1] has proved that any Jordan triple derivation on 2-torsion free semiprime ring is a derivation. A classical result of Herstein [2] asserts that any Jordan derivation on a 2-torsion free prime ring is a derivation. A brief proof of Herstein's result can be found Bresar M et al., [3]. Cusak [4] studied Jordan derivations on prime rings. Zalar [5] has proved that any left Jordan centralizer on a 2-torsion free semiprime ring is a left centralizer. Recently, Jing and $\mathrm{Lu}$ [6] introduced a concept of generalized Jordan derivation and generalized Jordan triple derivation. Vukman and kosi-Ulbl [7] studied an equation related to centralizers in semiprime rings. Molnar [8] studied on centralizers of an $\mathrm{H}^{*}$-algebra. Subba Reddy et al. [9-12] studied left multiplicative generalized derivations in prime and semiprime rings. Vukman [13] studied a note on generalized derivations of semiprime rings. In this paper, we can extended some results on left multiplicative generalized Jordan derivations of semiprime rings.

\section{Theorem 1}

Let $R$ be a 2-torsion free semiprime ring and let $F: R \rightarrow R$ be a left multiplicative generalized Jordan derivation. Then prove that $F$ is a left multiplicative generalized derivation.

Proof: We have therefore the relation,

$F\left(x^{2}\right)=x F(x)+D(x) x$, for all $x \in R$.

Here $D$ is a Jordan derivation on $R$.

Since $R$ is a semiprime ring one can conclude that $D$ is a derivation.

Let us denote $F-D$ by $T$.

Then we have, $T\left(x^{2}\right)=F\left(x^{2}\right)-D\left(x^{2}\right)$

$=x F(x)+D(x) x-D(x) x-x D(x)$

$=x F(x)-x D(x)$

$=x(F(x)-D(x))$

$T\left(x^{2}\right)=x T(x)$.

We have, therefore $T\left(x^{2}\right)=x T(x)$, for all $x \in R$. In other words, $T$ is a right Jordan centralizer of $R$. Since $R$ is a 2-torsion free semiprime ring. One can conclude that $T$ is a right centralizer in ref. [5]. Hence $F$ is of the form $F=D+T$. Where $D$ is a derivation and $T$ is a right centralizer of $R$. This means that $\mathrm{F}$ is a left multiplicative generalized derivation.

\section{Theorem 2}

Let $R$ be a 2-torsion free semiprime ring and let $F: R \rightarrow R$ be a left multiplicative generalized Jordan triple derivation. Then prove that $F$ is a left multiplicative generalized derivation.

Proof: We have therefore the relation,

$F(x y x)=x y F(x)+D(x) y x+x D(y) x$, for all $x, y \in R$.

where $D$ is a Jordan triple derivation of $R$.

Since $R$ is a semiprime ring one can conclude that, $D$ is a derivation by theorem $\mathrm{A}$ in ref. [1].

Let us denote $F-D$ by $T$.

*Corresponding author: Reddy CJS, Department of Mathematics, SV University, Tirupati-517502, Andhra Pradesh, India, Tel: +91 9441166259; E-mail: cjsreddysvu@gmail.com

Received January 02, 2018; Accepted February 27, 2018; Published March 06 2018

Citation: Reddy CJS, Nagesh K, Kumar AS (2018) Left Multiplicative Generalized Jordan Derivations of Semiprime Rings. J Generalized Lie Theory Appl 12: 288 doi: $10.4172 / 1736-4337.1000288$

Copyright: @ 2018 Reddy CJS, et al. This is an open-access article distributed under the terms of the Creative Commons Attribution License, which permits unrestricted use, distribution, and reproduction in any medium, provided the original author and source are credited. 
We have $T(x y x)=F(x y x)-D(x y x)$

$=x y F(x)+D(x) y x+x D(y) x-D(x) y x-x D(y) x-x y D(x)$.

$=x y F(x)-x y D(x)$

$=x y(F(x)-D(x))$

$T(x y x)=x y T(x)$.

We have therefore $T(x y x)=x y T(x)$, for all $x, y \in R$.

\section{Conclusion}

By theorem in ref. [14] one can conclude that $T$ is a right centralizer. We proved that $F$ can be written as $F=D+T$, where $D$ is a derivation and $T$ is a right centralizer, which means that $F$ is a left multiplicative generalized derivation.

Example: The following example express as a centralizer of a ring $R$ is both left and right centralizer of a additive mapping $T$, i.e., $T(x y)=T(x) y=x T(y)$, for all $x, y \in R$.

Consider the ring:

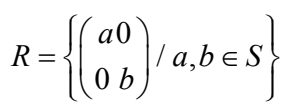

Where $S$ is any ring. Define $T: R \rightarrow R$, by

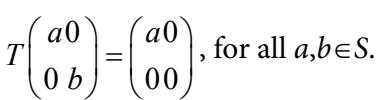

We can show that $T$ is a centralizer.

Let $x=\left(\begin{array}{cc}a_{1} & 0 \\ 0 & b_{1}\end{array}\right), y=\left(\begin{array}{cc}a_{2} & 0 \\ 0 & b_{2}\end{array}\right)$, where $x, y \in R$ and $a_{1}, b_{1}, a_{2}, b_{2} \in S$.

Applying $T$, we find that:

$T(x y)=\left(\begin{array}{cc}a_{1} a_{2} 0 \\ 0 & 0\end{array}\right)=T(x) y=x T(y)$.

Thus, we obtain $T$ is a centralizer.

\section{References}

1. Bresar M (1989) Jordan mappings of semiprime rings. J Algebra 127: 218-228.

2. Herstein IN (1957) Jordan derivations of prime rings. Proc Amer Math Soc 8: 1104-1119.

3. Bresar M, Vukman J (1988) Jordan derivations on prime rings. Bull Austral Math Soc 37: 321-322.

4. Cusak J (1975) Jordan derivations on rings. Proc Amer Math Soc 53: 321-324

5. Zalar B (1991) On centralizers of semiprime rings. Comment Math Univ Caro 32: 609-614.

6. Jing W, Lu S (2003) Generalized Jordan derivations on prime rings and standard operator Algebras. Taiwanese J Math 7: 605-613.

7. Vukman J, Kosi-Ulbl I (2003) An equation related to centralizers in semiprime rings. Glasnik Mat 38: 253-261.

8. Molnar L (1995) On centralizers of an $\mathrm{H}^{*}$ - algebra. Publ Math Debrecen 46 89-95.

9. Subba Reddy CJ, Vijay Kumar V, Mallikarjuna Rao S (2015) Left multiplicative generalized derivations on right ideal in semiprime rings. IOSR Journal of Mathematics 11: 60-63.

10. Subba Reddy CJ, Vijay Kumar V, Mallikarjuna Rao S (2015) Left multiplicative generalized derivations in semiprime rings. International Journal of Scientific Innovative Mathematical Research 3: 575-579.

11. Subba Reddy CJ, Mallikarjuna Rao S, Mahesh Kumar T (2014) Left multiplicative generalized derivations on semiprime rings. Mathematical Sciences International Research Journal 3: 859-861.

12. Subba Reddy CJ, Hemavathi K (2014) Left multiplicative generalized derivation on lie ideals in prime rings. International Journal of Mathematics Comutation 2: $12-18$.

13. Vukman J (2007) A note on generalized derivations of semiprime rings. Taiwanese Journal of Mathematics 11: 367-370.

14. Vukman J, Kosi-Ulbl I. A remark of a paper of L. Molnar. Publ Math Debrecen (In Press). 